\title{
QUALIDADE DE APLICAÇÃO DE HERBICIDA EM LAVOURA DE TRIGO
}

\author{
JORGE M. SUGUISAWA ${ }^{1}$, FABIANA N. FRANCO ${ }^{2}$, SAULO S. S. SILVA ${ }^{3}$, \\ AFONSO PECHE FILHO ${ }^{4}$
}

\begin{abstract}
RESUMO: O objetivo deste trabalho foi analisar a operação de aplicação de herbicida em lavoura de trigo utilizando ferramentas estatísticas da qualidade e tecnologia de sistema de informação geográfica, sendo os dados coletados em propriedade agrícola situada no Paraná. Foram considerados dois indicadores: percentagem de cobertura de gotas e densidade de gotas. Foram utilizadas, para avaliação dos resultados, técnicas da estatística descritiva, de controle de qualidade e do sistema de informação geográfica. Os resultados mostram que o processo de aplicação de defensivo avaliado apresenta irregularidade e grande variabilidade, necessitando de melhorias. Entretanto, tendo em vista a característica dos produtos utilizados (sistêmicos), pode-se considerar como razoável a qualidade da operação. A associação de técnicas de análises, como os da carta de controle, histograma de freqüência e da tecnologia SIG, permite boa caracterização do processo de pulverização empregado.
\end{abstract}

PALAVRAS-CHAVE: avaliação, pulverização, variabilidade.

\section{HERBICIDE SPRAYING QUALITY IN WHEAT CROP}

ABSTRACT: This work aimed to evaluate the pesticide spraying in no tillage system using statistical quality and GIS tools. The data were collected in a farm Paraná State, Brazil. Two indicators were considered: drop coverage percentage and drop density. Tools from descriptive statistics, quality control and GIS were used in the results evaluation. The spraying process presented irregularity and high variability, demanding improvement. However, considering the characteristics of the applied pesticides (systemics), the operation could be considered as reasonable. The association of the control chart, histogram and GIS is a tool which allowed the process characterization to be done.

KEYWORDS: evaluation, spraying, variability.

\section{INTRODUÇÃO}

Dentre as várias etapas que constituem o processo de produção agrícola, a aplicação de defensivos é uma das mais exigentes, pois deve atender não somente ao tratamento da área cultivada, mas também a cuidados com a preservação do ambiente (CHRISTOFOLETTI, 1999).

A eficiência de herbicidas é influenciada pela temperatura e umidade relativa do ar que afetam a absorção e a translocação desses compostos na planta. Com a diminuição da umidade relativa e/ou com o aumento da temperatura, as gotas da pulverização secam mais rapidamente, e a absorção do produto diminui ou, até mesmo, cessa, afetando o desempenho biológico. Segundo ROMAN (1999), a eficiência dos herbicidas no controle de plantas daninhas depende da quantidade de água usada como veículo em sua aplicação, da pressão de regulagem e da velocidade de deslocamento do pulverizador.

$\mathrm{Na}$ aplicação de agrotóxicos, é possível monitorar a qualidade operacional selecionando indicadores de desempenho que permitam coletar dados no campo e aferição de conformidades de

\footnotetext{
${ }^{1}$ Engoㅡ Agrícola, Mestre em Agronomia, Consultor, Itapeva - SP, jmsuguis@ yahoo.com.br

${ }^{2}$ Eng ${ }^{a}$ Agrônoma, Mestre em Agronomia, fabiana_nicoleti@aia.ajinomoto.com

${ }^{3}$ Eng ${ }^{0}$ Agrícola, Mestre em Agronomia, saulo_salaber@yahoo.com.br

${ }^{4}$ Engo Agrônomo, Mestre em Engenharia Agrícola, Pesquisador Científico do Centro APTA de Engenharia e Automação, IAC, Jundiaí -SP, peche@iac.sp.gov 
acordo com a prescrição técnica estabelecida. A qualidade de aplicação de agrotóxico pode ser crucial para atingir a produtividade almejada da cultura, e essa operação tem como principal objetivo aplicar a dose correta do defensivo no alvo desejado. Uma pulverização de baixa qualidade pode trazer como consequiências perdas significativas na produtividade, ocasionadas por reincidências de doenças, pragas e plantas daninhas na área (GADANHA JÚNIOR, 2000). Portanto, o monitoramento da qualidade da aplicação de defensivos agrícolas é muito importante para que se consiga um eficiente controle dos fatores danosos à cultura (BAIO, 2001).

Os conceitos de qualidade utilizados nas indústrias estão sendo adaptados à agricultura e são altamente aplicáveis, uma vez que as operações agrícolas são realizadas em cadeia, e o comprometimento da qualidade, em qualquer etapa, afeta as subseqüentes. O conceito de qualidade, sob o ponto de vista agronômico, é a realização de operações agrícolas ou a obtenção de produtos que estejam adequados às especificações ou aos padrões recomendados (DODSON, 1998). PECHE FILHO (1994) considera o controle de qualidade em operações agrícolas como a adoção de um conjunto de procedimentos que promovam serviços e resultados, atendendo, com precisão, às exigências da máquina, à qual cabe a operação seguinte, uma vez que a ineficiência de uma operação pode comprometer a operação subseqüente e, assim, sucessivamente.

Segundo BONILLA (1994), o controle de qualidade é perfeitamente adaptável ao sistema de produção agrícola, o qual, com a correção e a eliminação de desperdícios e falhas, redução de custos e aumento da produtividade, trará vantagens numerosas na competitividade do campo num futuro próximo. De acordo com essa linha de raciocínio, fica evidente que a sobrevivência das empresas agrícolas está intimamente ligada à implantação de novas técnicas administrativas com enfoque no Controle Estatístico de Processos (CEP), como destaca MILAN (1997).

O CEP, de acordo com MILAN \& FERNANDES (2002), constitui-se de ferramentas que permitem a melhoria da qualidade e da produtividade nas empresas. MONTGOMERY (1991) cita o CEP como um conjunto de técnicas úteis para resolução de problemas, alcance da estabilidade do processo e aumento da capacidade por meio da redução da variabilidade. Para BONILLA (1995), a utilização de ferramentas estatísticas de controle da qualidade possibilita detectar variações e oscilações nas operações agrícolas, a tempo de minimizar a possibilidade de sua concretização fora dos padrões especificados para o processo.

Vários autores têm estudado a utilização de técnicas estatísticas de controle de qualidade em operações agrícolas mecanizadas nas mais diversas culturas, sendo o milho a cultura de maior destaque nessa linha de pesquisa. PASQUA et al. (1996) utilizaram técnicas estatísticas de controle de qualidade nas operações de semeadura, adubação de cobertura e pulverização. Na pulverização, o indicador selecionado para a avaliação da operação foi a regularidade de vazão de líquido pelos bicos utilizados na operação. Os autores constataram que o processo não se encontrava sob controle estatístico e que o mesmo não atendeu à especificação determinada pelo fabricante.

Nesse enfoque, o objetivo do presente trabalho foi analisar a qualidade operacional da aplicação de herbicida em lavoura de trigo focando a variação da densidade e cobertura de gotas.

\section{MATERIAL E MÉTODOS}

O trabalho foi conduzido em uma propriedade agrícola situada no município de Piraí do Sul PR, cultivada com 53 ha de trigo em relevo suave ondulado. A pulverização foi realizada com pulverizador de barra, com assistência de ar, da marca Jacto, modelo Advance Vortex, com 37 bicos Jacto, tipo $110^{\circ}$-AVI-02. A aplicação do herbicida Glifosato ${ }^{\circledR}$ visou a controlar plantas indesejáveis que pudessem prejudicar a emergência e o desenvolvimento inicial do trigo, sendo de ação sistêmica. O pulverizador foi regulado para aplicar um volume de calda de $120 \mathrm{~L} \mathrm{ha}^{-1}$ à pressão de trabalho de $310 \mathrm{kPa}$, espaçamento entre bicos de $0,50 \mathrm{~m}$, altura da barra de pulverização de $0,50 \mathrm{~m}$ do alvo e velocidade de trabalho de $8 \mathrm{~km} \mathrm{~h}^{-1}$.

Os indicadores avaliados foram: densidade de gotas e percentagem de cobertura de gotas, distribuindo-se sobre o solo, em 17 pontos de amostragem aleatória, cinco cartões hidrossensíveis 
de forma paralela à barra de pulverização (Figura 1). Esses pontos foram georreferenciados, com a finalidade de construir mapas temáticos.

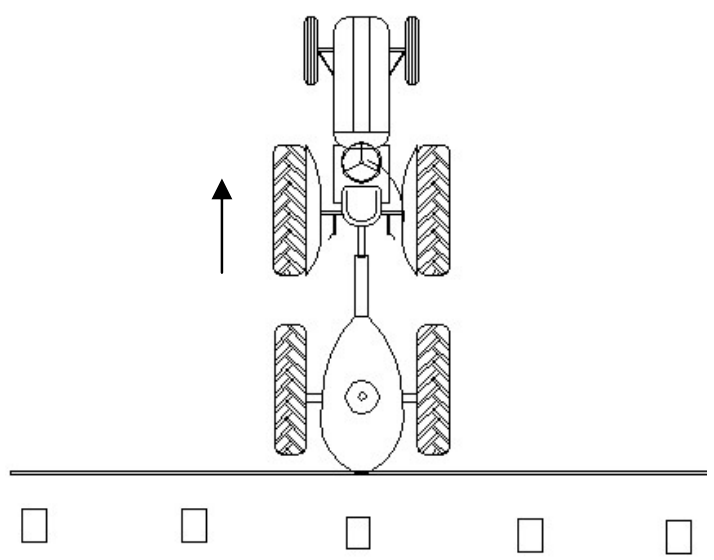

FIGURA 1. Esquema de distribuição dos cartões sensíveis à água, para avaliação da densidade e percentagem de cobertura de gotas.

Os papéis hidrossensíveis, após a passagem do pulverizador, foram recolhidos, codificados e embalados em plástico para evitar contaminação pela umidade do ar. Posteriormente, para análise de percentagem de cobertura de gotas, foram copiados no Departamento de Engenharia Rural da ESALQ/USP, com auxílio de scanner com resolução de 600 dpi. Os arquivos obtidos foram analisados pelo software IDRISI for Windows ${ }^{\circledR}$. Já para a análise de densidade de gotas, utilizou-se de lupa para contagem manual de gotas em área de $1 \mathrm{~cm}^{2}$.

Para a avaliação da qualidade dessa operação, utilizou-se de EPI (equipamentos de proteção individual), papéis hidrossensíveis, GPS Garmin, modelo e-Trex Legend, Palm Compaq, modelo iPAQ H3850, softwares Field Rover II $^{\circledR}$, SSToolbox ${ }^{\circledR}$ e IDRISI for Windows ${ }^{\circledR}$ para avaliar a percentagem de cobertura das gotas.

Os métodos estatísticos utilizados na análise da variabilidade foram: medidas de dispersão e de tendência central, histogramas e cartas de controle por variáveis, ferramentas essas empregadas no controle estatístico de processo (CEP), citadas por RYAN (1989) e DELLARETTI FILHO \& DRUMOND (1994).

\section{RESULTADOS E DISCUSSÃO}

Na Tabela 1, são apresentados os resultados referentes à análise estatística de variabilidade da percentagem de cobertura de gotas e densidade de gotas com base em parâmetros descritivos da operação de aplicação de herbicidas.

TABELA 1. Resultado da análise da estatística descritiva dos indicadores avaliados na operação de aplicação de herbicidas.

\begin{tabular}{lcc}
\hline \multirow{2}{*}{ Descrição } & \multicolumn{2}{c}{ Indicadores } \\
\cline { 2 - 3 } & Cobertura de Gotas (\%) & Densidade $\left(\right.$ gotas cm $\left.^{-2}\right)$ \\
\hline Média & 34,45 & 162,31 \\
Mediana & 32,31 & 141,00 \\
Moda & 32,00 & 141,00 \\
Desvio-padrão & 13,59 & 66,04 \\
C.V.(\%) & 39,44 & 40,69 \\
Curtose & 1,76 & 2,78 \\
Assimetria & 1,14 & 1,45 \\
Amplitude & 67,85 & 381,00 \\
Mínimo & 9,65 & 28,00 \\
Máximo & 77,50 & 409,00 \\
\hline
\end{tabular}


A análise dos resultados referente à cobertura de gotas mostra aplicação relativamente irregular, apesar de o coeficiente de curtose ser positivo, moda e mediana muito próximas, o que indica muitos pontos em torno da média, mas a amplitude é alta; os dados partem de um valor 3,5 vezes menor que a média e chegam a valores 2,24 maiores, influenciando no valor do coeficiente de variação de $39,44 \%$ considerado muito alto para esse tipo de operação, e no alto desvio-padrão correspondente a quase $1 / 3$ do valor da média. $O$ coeficiente de assimetria positivo indica leve tendência de a percentagem de cobertura aumentar em alguns pontos da área, que equivale a dizer que, em alguns pontos, a cobertura do alvo foi bastante acentuada, enquanto, em outros, poucas gotas atingiram o alvo. Na Figura 2, apresentam-se o histograma e a curva normal da percentagem de cobertura de gotas, na qual é possível observar a assimetria positiva em função de influência dos valores acima da média, deslocando a curva levemente para a direita.

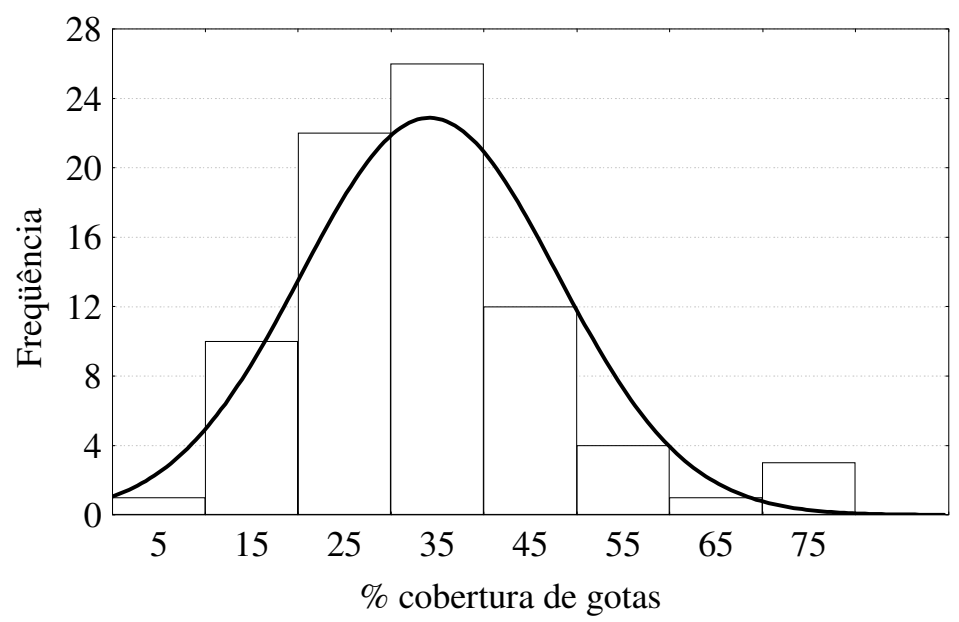

FIGURA 2. Histograma de distribuição e curva normal da percentagem de cobertura de gotas.

Na Figura 3, é apresentado o gráfico da distribuição da percentagem de cobertura de gotas, podendo-se avaliar a variabilidade ocorrente nessa operação; observa-se que $8 \%$ dos pontos amostrados se encontravam com percentual de cobertura menor que 23 , enquanto, no outro extremo, $4 \%$ dos pontos tiveram percentual de cobertura maior que 64. Pode-se observar, também, que percentual de cobertura maior que 50 foi observado em apenas $14 \%$ dos pontos amostrados.

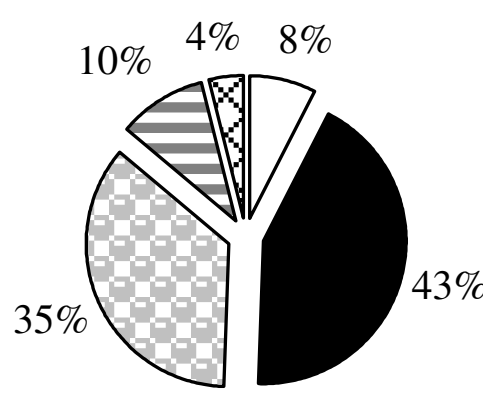

$\%$ Cobertura
$\square<23$

$0>64$

FIGURA 3. Distribuição percentual de cobertura de gotas.

Na Figura 4(a), pode-se observar pela carta de controle que a operação apresentou tendência descendente; essa tendência pode ser explicada pela elevação da temperatura ambiente e pela diminuição da umidade relativa, dado não mensurado no estudo. $\mathrm{O}$ relevo ondulado pode ter influenciado nas amostras 13 e 14, que atingiram valores acima do LSC (45\%), sofrendo influência da altura da barra do pulverizador, o que acarretou maior cobertura de gotas nas mesmas. Em relação à carta de amplitude de percentagem de cobertura de gotas, observa-se, na Figura 4(b), que apenas a amostra 14 se encontrou fora do LSC. 

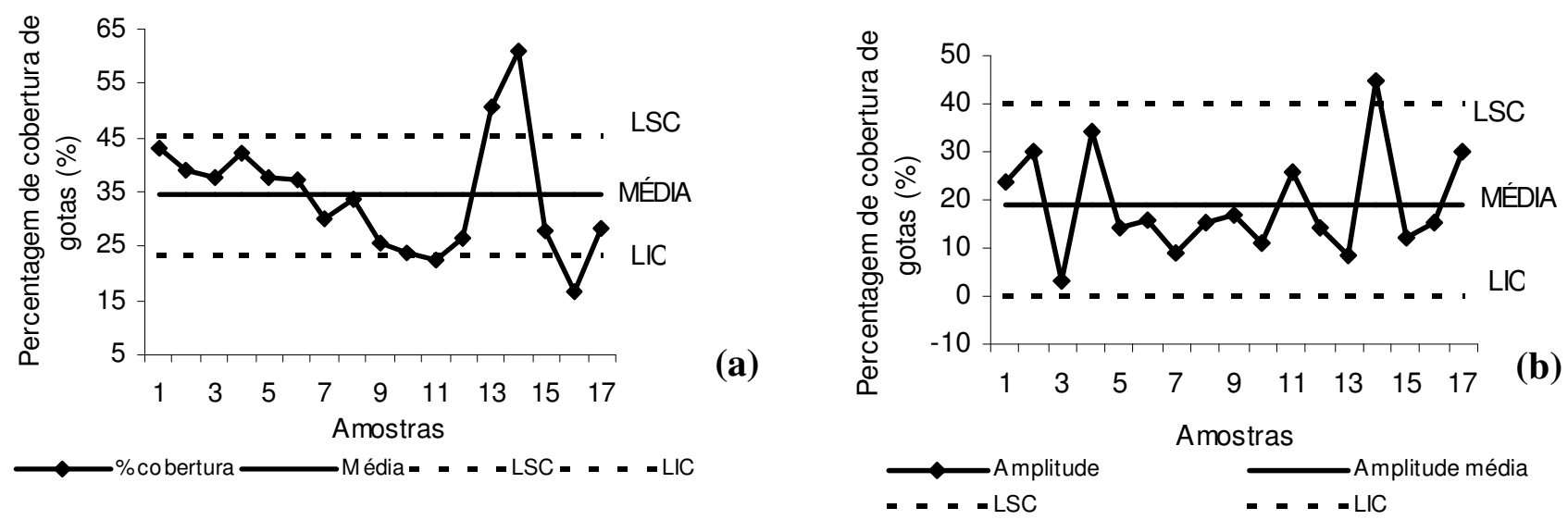

FIGURA 4. (a) Carta de controle das médias de percentagem de cobertura de gotas. (b) Carta de controle das amplitudes da percentagem de cobertura de gotas.

Com relação à densidade de gotas, a análise mostra novamente deposição relativamente irregular, devido às diferenças entre as medidas de tendência central (média, mediana e moda), demonstrando, também, tendência de a maioria dos dados apresentarem menor valor de densidade de gotas e que existiram diferenças significativas entre os resultados obtidos, o que pode ser comprovado pelo alto valor do desvio-padrão e do coeficiente de variação. Ocorreu alta variabilidade para esse indicador, fato confirmado pela amplitude, que apresentou valor próximo ao valor máximo, ou seja, a área apresentou pontos em que o alvo foi atingido por baixa densidade e pontos onde o alvo foi coberto com elevada densidade.

Na Figura 5, verificou-se, no histograma da densidade de gotas da operação de pulverização, assimetria positiva, sem tendência à normalidade, com valores bastante variáveis, sendo que os extremos não representaram a maioria dos dados, que se concentraram na faixa de 125 gotas $\mathrm{cm}^{-2}$.

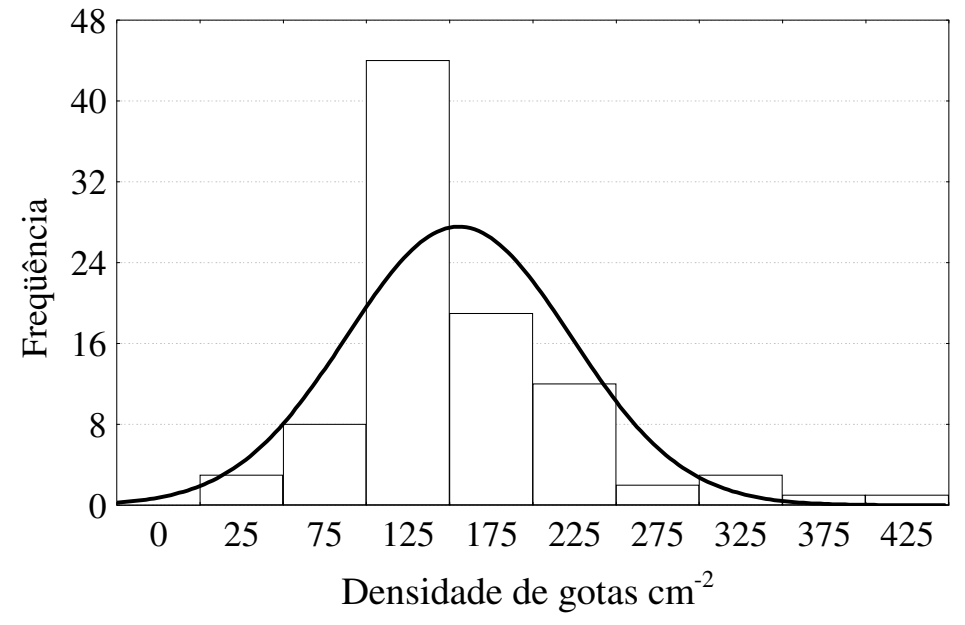

FIGURA 5. Histograma de distribuição e curva normal da densidade de gotas.

Pode-se observar, na Figura 6, que 55\% dos dados se encontram próximos à média e que valores considerados extremos correspondem a apenas $6 \%$ das observações. 


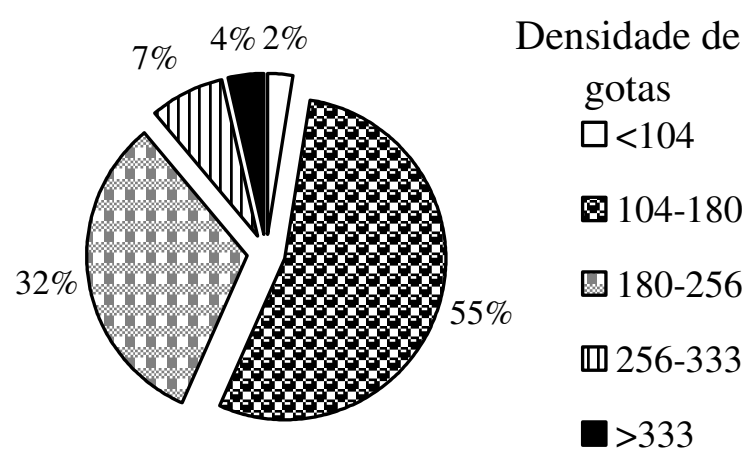

FIGURA 6. Distribuição percentual da densidade de gotas.

Observa-se, na Figura 7, o mapa da percentagem de cobertura de gotas, no qual houve maior concentração desse indicador do lado direito do mapa e alta concentração de valores abaixo da média do lado esquerdo. A explicação para esse comportamento pode estar no aumento da temperatura ambiente e na diminuição da umidade relativa do ar no decorrer do dia, uma vez que a operação se iniciou da direita do mapa para a esquerda.
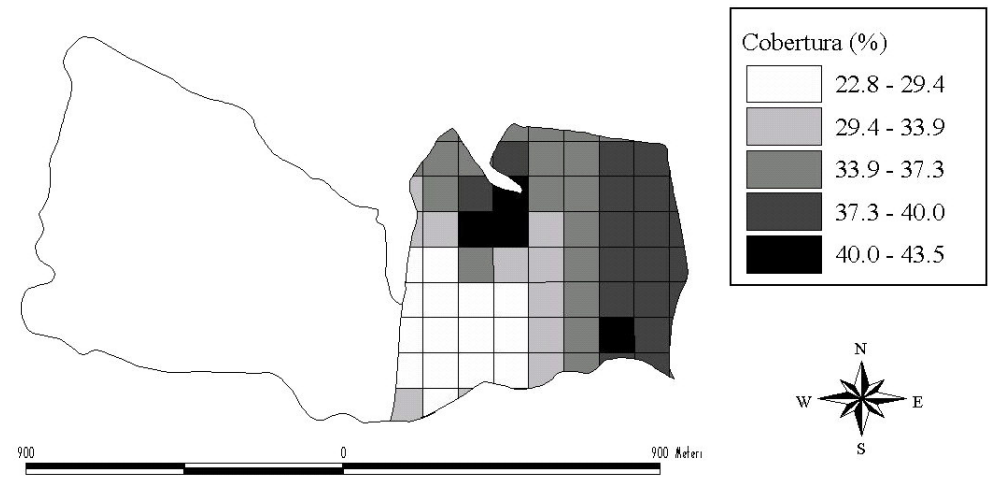

FIGURA 7. Mapa da distribuição espacial da percentagem de cobertura de gotas.

Na Figura 8, é possível observar o mapa gerado pela interpolação dos dados de densidade de gotas. Houve menor concentração de densidade de gotas do lado direito superior do mapa e alta concentração de valores acima da média do lado esquerdo superior. A porção inferior caracteriza-se por homogeneidade de amostras com valores de densidade de gotas superior à média.
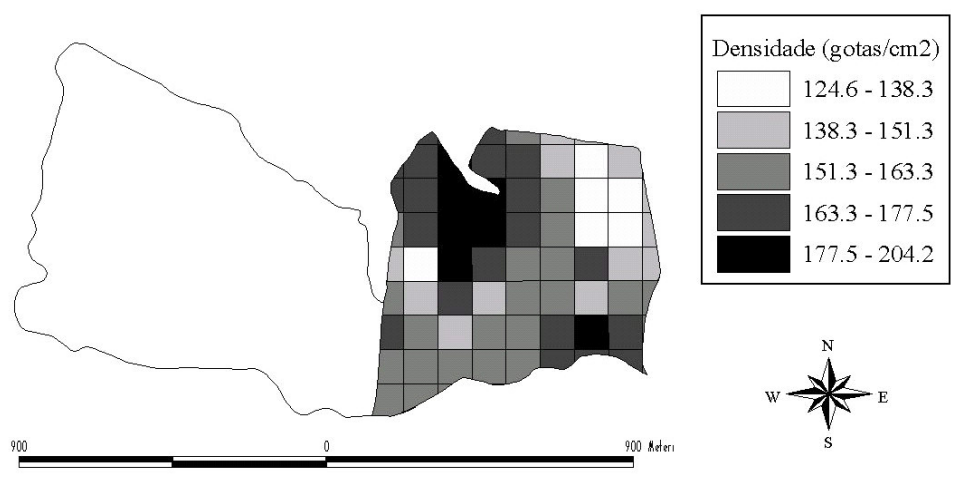

FIGURA 8. Mapa da distribuição espacial da densidade de gotas.

\section{CONCLUSÕES}

O processo de aplicação de defensivo avaliado apresenta irregularidade e grande variabilidade, necessitando de melhorias. Entretanto, tendo em vista a característica dos produtos 
utilizados (sistêmicos), pôde-se considerar como razoável a qualidade da operação. A associação da carta de controle, do histograma e do SIG permitiram caracterização da variabilidade operacional e, conseqüentemente, podem ser consideradas eficientes como ferramentas para análise da qualidade do processo de pulverização de herbicidas.

\section{AGRADECIMENTOS}

À Fazenda Paiquerê - PR, e ao Centro Apta de Engenharia e Automação (CAPTEA) - IAC, Jundiaí - SP.

\section{REFERÊNCIAS}

BAIO, F.H.R. Aplicação de defensivos baseada na variabilidade espacial de plantas daninhas. 2001. 113 f. Dissertação (Mestrado em Máquinas Agrícolas) - Escola Superior de Agricultura "Luiz de Queiroz", Universidade de São Paulo, Piracicaba, 2001.

BONILLA, J.A. Métodos quantitativos para qualidade total na agricultura. 2.ed. Contagem: Littera Maciel, 1995. 250 p.

BONILLA, J.A. Qualidade total na agricultura: fundamentos e aplicações. 2.ed. Belo Horizonte: Centro de Estudos de Qualidade Total na Agricultura, 1994. 344 p.

CHRISTOFOLETTI, J.C. Considerações sobre tecnologia de aplicação de defensivos agrícolas. Boletim Técnico, São Paulo, n.5, jun. 1999. Disponível em: <www.teejet.com.br>. Acesso em: 26 ago. 2003

DELLARETTI FILHO, O.; DRUMOND, F.B. Itens de controle e avaliação de processos. Belo Horizonte: Fundação Christiano Ottoni, 1994. 151 p.

DODSON, M.S. Avaliação da influência de indicadores de qualidade no custo operacional de um sistema de produção de milho (Zea mays L.): estudo de caso de semeadura. 1998. 80 f. Monografia (Trabalho de Graduação) - Faculdade de Ciências Agrárias e Veterinárias, Universidade Estadual Paulista, Jaboticabal, 1998.

GADANHA JÚNIOR, C.D. Avaliação do tempo de resposta de controladores eletrônicos em pulverizadores agrícolas. 2000. 125 f. Tese (Doutorado em Máquinas Agrícolas) - Faculdade de Ciências Agronômicas, Universidade Estadual Paulista, Botucatu, 2000.

MILAN, M. Controle de qualidade em operações agrícolas. NOTESALQ, Piracicaba, v.5, n.11, p.4$5,1997$.

MILAN, M.; FERNANDES, R.A.T. Qualidade das operações de preparo de solo por controle estatístico de processo. Scientia Agrícola, Piracicaba, v.59, n.2, p.261-6, abr./jun., 2002.

MONTGOMERY, D.C. Introduction to statistical quality control. New York: John Wiley, 1991. $677 \mathrm{p}$.

PASQUA, S.E.; MILAN, M.; PECHE FILHO, A. Controle de qualidade em operações agrícolas mecanizadas na cultura do milho (Zea mays L.) In: CONGRESSO BRASILEIRO DE ENGENHARIA AGRÍCOLA, 25., 1996, Bauru. Anais... Bauru: Sociedade Brasileira de Engenharia Agrícola, 1996. 1 CD-ROM.

PECHE FILHO, A. Qualidade total na agricultura. In: SIMPÓSIO DE QUALIDADE TOTAL NA AGRICULTURA, 1., 1994, São Paulo. Anais... São Paulo: AEASP, 1994. p.19-35.

ROMAN, E.S. Tecnologia de aplicação de herbicida na dessecação de Brachiaria plantaginea (Link) Hitchc. Passo Fundo: Embrapa Trigo, 1999. 7 p. Disponível em:

<www.cnpt.embrapa.br/biblio/p_ci01.htm>. Acesso em: 10 set. 2006

RYAN, T.P. Statistical methods for quality improvement. New York: John Wiley, 1989. 446 p. 